ANNALES

POLONICI MATHEMATICI

LXXIX.2 (2002)

\title{
Interval oscillation criteria for second order self-adjoint matrix differential systems with damping
}

\author{
by QIGUi YANG (Guilin and Guangzhou)
}

\begin{abstract}
By using the generalized Riccati technique and the averaging technique, we establish new oscillation criteria for the second order self-adjoint matrix differential system with damping

$$
\left(P(t) Y^{\prime}(t)\right)^{\prime}+r(t) P(t) Y^{\prime}(t)+Q(t) Y(t)=0, \quad t \geq t_{0} .
$$

The criteria are different from most known ones in the sense that they are based on the information only on a sequence of subintervals of $\left[t_{0}, \infty\right)$, rather than on the whole half-line. In particular, our results complement a number of existing results and handle a case that is not covered by known criteria. Moreover, examples indicating the importance of our results are also included.
\end{abstract}

1. Introduction. Consider the second order matrix differential equation with damping

$$
\left(P(t) Y^{\prime}(t)\right)^{\prime}+r(t) P(t) Y^{\prime}(t)+Q(t) Y(t)=0, \quad t \geq t_{0},
$$

where $r(t) \in C\left(I=\left[t_{0}, \infty\right), \mathbb{R}\right)$ and $Y(t), P(t)$ and $Q(t)$ are $n \times n$ real continuous matrix functions with $P(t), Q(t)$ symmetric and $P(t)$ positive definite for $\left[t_{0}, \infty\right)\left(P(t)>0, t \geq t_{0}\right)$. A solution of the system (1.1) is said to be nontrivial if $\operatorname{det} Y(t) \neq 0$ for at least one $t \in\left[t_{0}, \infty\right)$, and a nontrivial solution $Y(t)$ of (1.1) is said to be prepared or self-conjugate if

$$
Y^{*}(t) P(t) Y^{\prime}(t)-\left[Y^{*}(t)\right]^{\prime} P(t) Y(t)=0, \quad t \geq t_{0},
$$

where for any matrix $A$, the transpose of $A$ is denoted by $A^{*}$. A prepared solution $Y(t)$ of the system (1.1) is called oscillatory on $\left[t_{0}, \infty\right)$ if its determinant vanishes somewhere in $[T, \infty)$ for each $T \geq t_{0}$, otherwise, it is called nonoscillatory. Finally, the system $(1.1)$ is called oscillatory on $\left[t_{0}, \infty\right)$ if every prepared solution is oscillatory.

2000 Mathematics Subject Classification: 34A30, 34C10.

Key words and phrases: interval oscillation, matrix differential system, self-adjoint.

Research supported by the Science Foundation of Guangxi Province of China. 
The oscillation problem for the matrix system (1.1) and less general systems

$$
\begin{gathered}
\left(P(t) Y^{\prime}(t)\right)^{\prime}+Q(t) Y(t)=0, \quad t \geq t_{0}, \\
\left.Y^{\prime \prime}(t)\right)+Q(t) Y(t)=0, \quad t \geq t_{0},
\end{gathered}
$$

and the corresponding scalar equations

$$
\begin{gathered}
\left(p(t) y^{\prime}(t)\right)^{\prime}+q(t) y(t)=0, \\
y^{\prime \prime}(t)+q(t) y(t)=0
\end{gathered}
$$

has been discussed by numerous authors and by different methods (see, for example, [1-16] or other references contained therein).

An important tool in the study of oscillatory behavior of solutions for (1.3)-(1.6) is the averaging technique which goes back as far as the classical papers of Wintner [15] and Hartman [5] giving sufficient oscillation conditions for (1.6). The result of Wintner was improved by Kamenev [7], and further extensions of Kamenev's criterion have been obtained by Philos [13] and for the matrix system (1.3) by Erbe, Kong and Ruan [4], Meng, Wang and Zhang [11], Kumari and Umamaheswaram [9] and Wang [14].

However, all the aforementioned papers involve $P(t)$ and the integral of $Q(t)$ and hence require the knowledge of $Q(t)$ on the entire half-line $\left[t_{0}, \infty\right)$. But, from the Sturm Separation Theorem, if there exists a sequence of subintervals $\left[a_{i}, b_{i}\right]$ of $\left[t_{0}, \infty\right)$, with $a_{i} \rightarrow \infty$, such that for each $i$ there exists a solution of (1.5) that has at least two zeros in $\left[a_{i}, b_{i}\right]$, then every solution of (1.5) is oscillatory, no matter how "bad" (1.5) is (or $P$ and $Q$ are) on the remaining part of $\left[t_{0}, \infty\right)$. Motivated by this surprising result, we further study the system (1.1).

The purpose of this paper is to obtain interval oscillation criteria for the system (1.1) making use of the technique similar to that exploited by Philos [13] and Kong [8] for the second order linear ordinary differential equations. New interval oscillation criteria established for the second order linear matrix differential system (1.1) are different from most known ones in the sense that they are based on the information only on a sequence of subintervals of $\left[t_{0}, \infty\right)$, rather than on the whole half-line. Our results involve a Kamenev type condition and improve and extend the results of Erbe, Kong and Ruan [4], Wang [14], Huang [6], Kamenev [7], Kong [8] and Philos [13]; they also complement a number of other existing results and handle cases which are not covered by known criteria in [1-16] and other papers.

In this paper, by using the generalized Riccati technique and the averaging technique and by considering the function $H(t, s) k(s)$ which may not have a nonpositive partial derivative on $D_{0}=\left\{(t, s): t>s \geq t_{0}\right\}$ with respect to the second variable (the assumption $\partial H(t, s) / \partial s \leq 0$ on $D_{0}$ appears e.g. in $[4,5,15])$, we obtain new general oscillation criteria for the 
system (1.1), that is, criteria given by the behavior of (1.1) (or of $P(t)$ and $Q(t))$ only on a sequence of subintervals of $\left[t_{0}, \infty\right)$. By choosing appropriate functions $H, k$ and $v$, we present a series of explicit oscillation criteria.

Finally, we include an example of a system whose oscillation cannot be proved by the previously known criteria.

Hereafter we denote the trace of an $n \times n$ matrix $A$ by $\operatorname{tr}(A)$. Further, $E_{n}$ is the $n \times n$ identity matrix, and the eigenvalues of the $n \times n$ symmetric matrix $A$ (in increasing order) are $\lambda_{\min }[A]=\lambda_{n}[A] \leq \ldots \leq \lambda_{1}[A]=\lambda_{\max }[A]$.

Define

$$
D_{0}=\left\{(t, s): t>s \geq t_{0}\right\}, \quad D=\left\{(t, s): t \geq s \geq t_{0}\right\} .
$$

2. Oscillation results. Our main results are the following theorems and corollaries.

TheOrEm 2.1. Let functions $H \in C(D, \mathbb{R}), h_{1}, h_{2} \in C\left(D_{0}, \mathbb{R}\right)$ and $k, v \in$ $C^{1}\left(\left[t_{0}, \infty\right),(0, \infty)\right)$ satisfy the following conditions:

$$
\begin{aligned}
& H(t, t)=0 \text { for } t \geq t_{0}, H(t, s)>0 \text { on } D_{0} \\
& \frac{\partial}{\partial t}(H(t, s) k(t))-\left(r(t)-\frac{v^{\prime}(t)}{v(t)}\right) H(t, s) k(t)=h_{1}(t, s), \forall(t, s) \in D_{0} \\
& \frac{\partial}{\partial s}(H(t, s) k(s))-\left(r(s)-\frac{v^{\prime}(s)}{v(s)}\right) H(t, s) k(s)=-h_{2}(t, s), \forall(t, s) \in D_{0} .
\end{aligned}
$$

Assume also that for each sufficiently large $T_{0} \geq t_{0}$, there exist $a, b, c \in \mathbb{R}$ with $T_{0} \leq a<c<b$ such that

$$
\begin{aligned}
& \frac{1}{H(c, a)} \lambda_{n}\left[\int_{a}^{c}\left\{H(s, a) k(s) v(s) Q(s)-\frac{1}{4} \frac{h_{1}^{2}(s, a)}{H(s, a) k(s)} v(s) P(s)\right\} d s\right] \\
& +\frac{1}{H(b, c)} \lambda_{1}\left[\int_{c}^{b}\left\{H(b, s) k(s) v(s) Q(s)-\frac{1}{4} \frac{h_{2}^{2}(b, s)}{H(b, s) k(s)} v(s) P(s)\right\} d s\right]>0 .
\end{aligned}
$$

Then the system (1.1) is oscillatory.

Proof. Suppose that there exists a prepared solution $Y(t)$ of the system (1.1) which is not oscillatory. Without loss of generality, we may assume that $\operatorname{det} Y(t) \neq 0$ for $t \geq t_{0}$. Define

$$
W(t)=v(t) P(t) Y^{\prime}(t) Y^{-1}(t) \quad \text { for } t \geq t_{0} .
$$

By differentiating the matrix (2.2) and making use of (1.1), we find that $W(t)$ satisfies the Riccati equation for $t \in\left[t_{0}, \infty\right)$ : 


$$
\begin{aligned}
W^{\prime}(t) & =\frac{v^{\prime}(t)}{v(t)} W(t)+v(t)\left\{\left[P(t) Y^{\prime}(t)\right]^{\prime} Y^{-1}(t)-P(t)\left[Y^{\prime}(t) Y^{-1}(t)\right]^{2}\right\} \\
& =\left(r(t)-\frac{v^{\prime}(t)}{v(t)}\right) W(t)-v(t) Q(t)-\frac{1}{v(t)} W(t) P^{-1}(t) W(t)
\end{aligned}
$$

that is,

$$
v(t) Q(t)=-W^{\prime}(t)-\left(r(t)-\frac{v^{\prime}(t)}{v(t)}\right) W(t)-\frac{1}{v(t)} W(t) P^{-1}(t) W(t) .
$$

On multiplying (2.3) (with $t$ replaced by $s$ ) by $H(t, s) k(s)$ and integrating with respect to $s$ from $c$ to $t$ for $t \in[c, b)$, we obtain

$$
\begin{aligned}
& \int_{c}^{t} H(t, s) k(s) v(s) Q(s) d s \\
& =-\int_{c}^{t} H(t, s) k(s) W^{\prime}(s) d s-\int_{c}^{t} H(t, s) k(s)\left(r(s)-\frac{v^{\prime}(s)}{v(s)}\right) W(s) d s \\
& \quad-\int_{c}^{t} H(t, s) k(s) \frac{1}{v(s)} W(s) P^{-1}(s) W(s) d s \\
& =H(t, c) k(c) W(c)-\int_{c}^{t} H(t, s) k(s) \frac{1}{v(s)} W(s) P^{-1}(s) W(s) d s \\
& \quad-\int_{c}^{t}\left[-\frac{\partial}{\partial s}(H(t, s) k(s))+\left(r(s)-\frac{v^{\prime}(s)}{v(s)}\right) H(t, s) k(s)\right] W(s) d s \\
& =H(t, c) k(c) W(c)-\int_{c}^{t} h_{2}(t, s) W(s) d s-\int_{c}^{t} H(t, s) \frac{k(s)}{v(s)} W(s) P^{-1}(s) W(s) d s .
\end{aligned}
$$

Since $P(t)>0$, we can let

$$
R(t)=\left[\frac{1}{v(t)} P^{-1}(t)\right]^{1 / 2}
$$

Substituting $R(t)$ into the above equation, we have

$$
\begin{aligned}
& \int_{c}^{t} H(t, s) k(s) v(s) Q(s) d s \\
& =H(t, c) k(c) W(c)-\int_{c}^{t} h_{2}(t, s) R^{-1}(s)[R(s) W(s) R(s)] R^{-1}(s) d s \\
& \quad-\int_{c}^{t} H(t, s) k(s) R^{-1}(s)[R(s) W(s) R(s)][R(s) W(s) R(s)] R^{-1}(s) d s
\end{aligned}
$$




$$
\begin{gathered}
=H(t, c) k(c) W(c)+\frac{1}{4} \int_{c}^{t} \frac{h_{2}^{2}(t, s)}{H(t, s) k(s)} v(s) P(s) d s \\
-\int_{c}^{t} R^{-1}(s)\left\{|H(t, s) k(s)|^{1 / 2}[R(s) W(s) R(s)]\right. \\
\left.+\frac{1}{2} \frac{h_{2}(t, s)}{|H(t, s) k(s)|^{1 / 2}} E_{n}\right\}^{2} R^{-1}(s) d s .
\end{gathered}
$$

Thus we obtain

$$
\int_{c}^{t}\left\{H(t, s) k(s) v(s) Q(s)-\frac{1}{4} \frac{h_{2}^{2}(t, s)}{H(t, s) k(s)} v(s) P(s)\right\} d s \leq H(t, c) k(c) W(c) .
$$

It follows that

$$
\begin{aligned}
\lambda_{1}\left[\int _ { c } ^ { t } \left\{H(t, s) k(s) v(s) Q(s)-\frac{1}{4} \frac{h_{2}^{2}(t, s)}{H(t, s) k(s)}\right.\right. & v(s) P(s)\} d s] \\
& \leq \lambda_{1}[H(t, c) k(c) W(c)] .
\end{aligned}
$$

Letting $t \rightarrow b^{-}$in (2.4) and dividing both sides by $H(b, c)$, we obtain

$$
\begin{array}{r}
\frac{1}{H(b, c)} \lambda_{1}\left[\int_{c}^{b}\left\{H(b, s) k(s) v(s) Q(s)-\frac{1}{4} \frac{h_{2}^{2}(b, s)}{H(b, s) k(s)} v(s) P(s)\right\} d s\right] \\
\leq \lambda_{1}[k(c) W(c)] .
\end{array}
$$

Similarly to the proof above, multiplying (2.3), with $t$ replaced by $s$, by $H(s, t) k(s)$ and integrating with respect to $s$ from $t$ to $c$ for $t \in(a, c]$, we obtain

$$
\begin{aligned}
\int_{t}^{c} H(s, t) k(s) & v(s) Q(s) d s \\
= & -\int_{t}^{c} H(s, t) k(s) W^{\prime}(s) d s-\int_{t}^{c} H(s, t) k(s)\left(r(s)-\frac{v^{\prime}(s)}{v(s)}\right) W(s) d s \\
& -\int_{t}^{c} H(s, t) k(s) \frac{1}{v(s)} W(s) P^{-1}(s) W(s) d s \\
= & -H(c, t) k(c) W(c)-\int_{t}^{c} H(t, s) k(s) \frac{1}{v(s)} W(s) P^{-1}(s) W(s) d s \\
& -\int_{t}^{c}\left[-\frac{\partial}{\partial s}(H(s, t) k(s))+H(s, t) k(s)\left(r(s)-\frac{v^{\prime}(s)}{v(s)}\right)\right] W(s) d s
\end{aligned}
$$




$$
\begin{aligned}
= & -H(c, t) k(c) W(c)+\int_{t}^{c} h_{1}(s, t) W(s) d s \\
& -\int_{t}^{c} H(s, t) \frac{k(s)}{v(s)} W(s) P^{-1}(s) W(s) d s .
\end{aligned}
$$

Since $P(t)>0$, we can again let $R(t)=\left[(1 / v(t)) P^{-1}(t)\right]^{1 / 2}$. Substituting $R(t)$ into the above equation, we obtain

$$
\begin{aligned}
\int_{t}^{c} H(s, t) k(s) v(s) Q(s) d s & \\
= & -H(c, t) k(s) W(c)+\int_{t}^{c} h_{1}(s, t) R^{-1}(s)[R(s) W(s) R(s)] R^{-1}(s) d s \\
& -\int_{t}^{c} H(s, t) k(s) R^{-1}(s)[R(s) W(s) R(s)][R(s) W(s) R(s)] R^{-1}(s) d s \\
= & -H(c, t) k(s) W(c)+\frac{1}{4} \int_{t}^{c} \frac{h_{1}^{2}(s, t)}{H(s, t) k(s)} v(s) P(s) d s \\
& -\int_{t}^{c} R{ }^{-1}(s)\left\{|H(s, t) k(s)|^{1 / 2}[R(s) W(s) R(s)]\right. \\
& \left.\quad+\frac{1}{2} \frac{h_{1}(s, t)}{|H(s, t) k(s)|^{1 / 2}} E_{n}\right\}^{2} R^{-1}(s) d s .
\end{aligned}
$$

Thus we obtain

$$
\int_{t}^{c}\left\{H(s, t) k(s) v(s) Q(s)-\frac{1}{4} \frac{h_{1}^{2}(s, t)}{H(s, t) k(s)} v(s) P(s)\right\} d s \leq-H(c, t) k(c) W(c) .
$$

Thus

$$
\begin{array}{r}
\lambda_{n}\left[\int_{t}^{c}\left\{H(s, t) k(s) v(s) Q(s)-\frac{1}{4} \frac{h_{1}^{2}(s, t)}{H(s, t) k(s)} v(s) P(s)\right\} d s\right] \\
\leq \lambda_{n}[-H(c, t) k(c) W(c)]=-\lambda_{1}[H(c, t) k(c) W(c)]
\end{array}
$$

where $t \in(a, c]$. Letting $t \rightarrow a^{+}$in the above inequality and dividing both sides by $H(c, a)$, we get

$$
\begin{array}{r}
\frac{1}{H(c, a)} \lambda_{n}\left[\int_{a}^{c}\left\{H(s, a) k(s) v(s) Q(s)-\frac{1}{4} \frac{h_{1}^{2}(s, a)}{H(s, a) k(s)} v(s) P(s)\right\} d s\right] \\
\leq-\lambda_{1}[k(c) W(c)] .
\end{array}
$$


Now we claim that $\operatorname{det} Y(t)$, where $Y(t)$ is any prepared solution of (1.1), has at least one zero in $(a, b)$.

Suppose the contrary. Adding (2.5) and (2.6), we have an inequality which contradicts the assumption (2.1). Thus, the conclusion holds.

Pick up a sequence $\left\{T_{i}\right\} \subset\left[t_{0}, \infty\right)$ such that $T_{i} \rightarrow \infty$ as $i \rightarrow \infty$. By the assumptions of Theorem 2.1, for each $i \in \mathbb{N}$, there exist $a_{i}, b_{i}, c_{i} \in \mathbb{R}$ such that $T_{i} \leq a_{i}<c_{i}<b_{i}$, and (2.1) holds with $a, b, c$ replaced by $a_{i}, b_{i}$, $c_{i}$, respectively. From the above claim, the determinant of every prepared solution $Y(t)$ has at least one zero $t_{i} \in\left(a_{i}, b_{i}\right)$. Noting that $t_{i}>a_{i} \geq T_{i}$, $i \in \mathbb{N}$, we see that $\operatorname{det} Y(t)$ has arbitrarily large zeros. Thus, the system (1.1) is oscillatory. The proof is complete.

Under a modification of the hypotheses of Theorem 2.1, we can obtain the following result.

CoROllary 2.1. Under the assumptions of Theorem 2.1 with (2.1) replaced by

$$
\begin{aligned}
\frac{1}{H(c, a)} \int_{a}^{c} H(s, a) k(s) v(s) & \operatorname{tr} Q(s) d s \\
& +\frac{1}{H(b, c)} \int_{c}^{b} H(b, s) k(s) v(s) \operatorname{tr} Q(s) d s \\
> & \frac{1}{4}\left(\frac{1}{H(c, a)} \int_{a}^{c} \frac{h_{1}^{2}(s, a) v(s)}{H(s, a) k(s)} \operatorname{tr} P(s) d s\right. \\
& \left.+\frac{1}{H(b, c)} \int_{c}^{b} \frac{h_{2}^{2}(b, s) v(s)}{H(b, s) k(s)} \operatorname{tr} P(s) d s\right)
\end{aligned}
$$

the system (1.1) is oscillatory.

The proof is similar to that of Theorem 2.1.

THEOREM 2.2. Under the assumptions of Theorem 2.1 with the condition including (2.1) replaced by:

$$
\limsup _{t \rightarrow \infty} \lambda_{n}\left[\int_{l}^{t}\left\{H(s, l) k(s) v(s) Q(s)-\frac{1}{4} \frac{h_{1}^{2}(s, l)}{H(s, l) k(s)} v(s) P(s)\right\} d s\right]>0
$$

and

$$
\limsup _{t \rightarrow \infty} \lambda_{1}\left[\int_{l}^{t}\left\{H(t, s) k(s) v(s) Q(s)-\frac{1}{4} \frac{h_{2}^{2}(t, s)}{H(t, s) k(s)} v(s) P(s)\right\} d s\right]>0,
$$

for each $l \geq t_{0}$, the system (1.1) is oscillatory.

Proof. For any $T \geq t_{0}$, let $a=T$. In (2.8) we choose $l=a$. Then there exists $c>a$ such that 


$$
\lambda_{n}\left[\int_{a}^{c}\left\{H(s, a) k(s) v(s) Q(s)-\frac{1}{4} \frac{h_{1}^{2}(s, a)}{H(s, a) k(s)} v(s) P(s)\right\} d s\right]>0,
$$

In (2.9) we choose $l=c$. Then there exists $b>c$ such that

$$
\lambda_{1}\left[\int_{c}^{b}\left\{H(b, s) k(s) v(s) Q(s)-\frac{1}{4} \frac{h_{2}^{2}(b, s)}{H(b, s) k(s)} v(s) P(s)\right\} d s\right]>0 .
$$

Combining (2.10) and (2.11) we obtain (2.1). The conclusion thus comes from Theorem 2.1.

COROLlaRY 2.2. Under the assumptions of Theorem 2.1 with the condition including (2.1) replaced by:

$$
\begin{aligned}
& \limsup _{t \rightarrow \infty} \int_{l}^{t}\left\{H(s, l) k(s) v(s) \operatorname{tr} Q(s)-\frac{1}{4} \frac{h_{1}^{2}(s, l)}{H(s, l) k(s)} v(s) \operatorname{tr} P(s)\right\} d s>0, \\
& \limsup _{t \rightarrow \infty} \int_{l}^{t}\left\{H(t, s) k(s) v(s) \operatorname{tr} Q(s)-\frac{1}{4} \frac{h_{2}^{2}(t, s)}{H(t, s) k(s)} v(s) \operatorname{tr} P(s)\right\} d s>0
\end{aligned}
$$

for each $l \geq t_{0}$, the system (1.1) is oscillatory.

If in Theorems 2.1-2.2 and Corollaries 2.1-2.2, $h_{1}(t, s)$ and $h_{2}(t, s)$ are replaced by $h_{1}(t, s) \sqrt{H(t, s) k(s)}$ and $h_{2}(t, s) \sqrt{H(t, s) k(s)}$ respectively, we can obtain the following results. The proofs are similar.

Theorem 2.3. Let $H \in C(D, \mathbb{R})$ satisfy condition (H1) in Theorem 2.1. Suppose that there exist $h_{1}, h_{2} \in C\left(D_{0}, \mathbb{R}\right)$ and $k, v \in C^{1}\left(\left[t_{0}, \infty\right),(0, \infty)\right)$ such that

$$
\begin{array}{rlrl}
\frac{\partial}{\partial t}(H(t, s) k(t))-H(t, s) k(t) & \left(r(t)-\frac{v^{\prime}(t)}{v(t)}\right) & & \\
& =h_{1}(t, s) \sqrt{H(t, s) k(t)}, & & \forall(t, s) \in D_{0} ; \\
\frac{\partial}{\partial s}(H(t, s) k(s))-H(t, s) k(s) & \left(r(s)-\frac{v^{\prime}(s)}{v(s)}\right) & & \\
= & -h_{2}(t, s) \sqrt{H(t, s) k(s)}, & \forall(t, s) \in D_{0} .
\end{array}
$$

Assume also that for each sufficiently large $T_{0} \geq t_{0}$, there exist $a, b, c \in \mathbb{R}$ with $T_{0} \leq a<c<b$ such that

$$
\begin{aligned}
& \frac{1}{H(c, a)} \lambda_{n}\left[\int_{a}^{c}\left\{H(s, a) k(s) v(s) Q(s)-\frac{1}{4} h_{1}^{2}(s, a) v(s) P(s)\right\} d s\right] \\
+ & \frac{1}{H(b, c)} \lambda_{1}\left[\int_{c}^{b}\left\{H(b, s) k(s) v(s) Q(s)-\frac{1}{4} h_{2}^{2}(b, s) v(s) P(s)\right\} d s\right]>0 .
\end{aligned}
$$

Then the system (1.1) is oscillatory. 
COROLlaRY 2.3. Under the assumptions of Theorem 2.3 with (2.14) replaced by

$$
\begin{aligned}
& \frac{1}{H(c, a)} \int_{a}^{c} H(s, a) k(s) v(s) \operatorname{tr} Q(s) d s \\
+ & \frac{1}{H(b, c)} \int_{c}^{b} H(b, s) k(s) v(s) \operatorname{tr} Q(s) d s \\
> & \frac{1}{4}\left(\frac{1}{H(c, a)} \int_{a}^{c} h_{1}^{2}(s, a) v(s) \operatorname{tr} P(s) d s+\frac{1}{H(b, c)} \int_{c}^{b} h_{2}^{2}(b, s) v(s) \operatorname{tr} P(s) d s\right),
\end{aligned}
$$

the system (1.1) is oscillatory.

THEOREM 2.4. Under the assumptions of Theorem 2.3 with the condition including (2.14) replaced by:

$$
\limsup _{t \rightarrow \infty} \lambda_{n}\left[\int_{l}^{t}\left\{H(s, l) k(s) v(s) Q(s)-\frac{1}{4} h_{1}^{2}(s, l) v(s) P(s)\right\} d s\right]>0
$$

and

$$
\limsup _{t \rightarrow \infty} \lambda_{1}\left[\int_{l}^{t}\left\{H(t, s) k(s) v(s) Q(s)-\frac{1}{4} h_{2}^{2}(t, s) v(s) P(s)\right\} d s\right]>0,
$$

for each $l \geq t_{0}$, the system (1.1) is oscillatory.

COROLlary 2.4. Under the assumptions of Theorem 2.3 with the condition including (2.14) replaced by:

$$
\limsup _{t \rightarrow \infty} \int_{l}^{t}\left\{H(s, l) k(s) v(s) \operatorname{tr} Q(s)-\frac{1}{4} h_{1}^{2}(s, l) v(s) \operatorname{tr} P(s)\right\} d s>0
$$

and

$$
\limsup _{t \rightarrow \infty} \int_{l}^{t}\left\{H(t, s) k(s) v(s) \operatorname{tr} Q(s)-\frac{1}{4} h_{2}^{2}(t, s) v(s) \operatorname{tr} P(s)\right\} d s>0
$$

for each $l \geq t_{0}$, the system (1.1) is oscillatory.

Moreover, let $k(t)=1$ and $H:=H(t-s)$ in Theorem 2.3. Then $\partial(H(t-s)) / \partial t=-\partial(H(t-s)) / \partial s$ and denote them by $h(t-s)$. Thus

$$
\begin{aligned}
& h_{1}(t, s)=\frac{h(t-s)}{\sqrt{H(t-s)}}-\sqrt{H(t-s)}\left(r(t)-\frac{v^{\prime}(t)}{v(t)}\right), \\
& h_{2}(t, s)=-\frac{h(t-s)}{\sqrt{H(t-s)}}-\sqrt{H(t-s)}\left(r(s)-\frac{v^{\prime}(s)}{v(s)}\right) .
\end{aligned}
$$

Applying Theorem 2.3 and Corollary 2.3, we obtain 
THEOREM 2.5. Under the assumptions of Theorem 2.3 with the condition including (2.14) replaced by: for any $T \geq t_{0}$, there exist $T \leq a<c$ such that

$$
\begin{aligned}
& \lambda_{n}\left[\int_{a}^{c}\{H(s-a) v(s) Q(s)\right. \\
& \left.\left.-\frac{1}{4}\left(\frac{h(s-a)}{\sqrt{H(s-a)}}-\sqrt{H(s-a)}\left(r(s)-\frac{v^{\prime}(s)}{v(s)}\right)\right)^{2} v(s) P(s)\right\} d s\right] \\
& +\lambda_{1}\left[\int_{a}^{c}\{H(s-a) v(2 c-s) Q(2 c-s)\right. \\
& -\frac{1}{4}\left(\frac{h(s-a)}{\sqrt{H(s-a)}}+\sqrt{H(s-a)}\left(r(2 c-s)-\frac{v^{\prime}(2 c-s)}{v(2 c-s)}\right)\right)^{2} \\
& \quad \times v(2 c-s) P(2 c-s)\} d s]>0,
\end{aligned}
$$

the system (1.1) is oscillatory.

Proof. Let $b=2 c-a$. Then $H(b-c)=H(c-a)=H((b-a) / 2)$, and for any $w \in L[a, b]$, we have

$$
\int_{c}^{b} w(s) d s=\int_{a}^{c} w(2 c-s) d s
$$

Hence

$$
\int_{c}^{b} H(b-s) v(s) Q(s) d s=\int_{a}^{c} H(s-a) v(2 c-s) Q(2 c-s) d s
$$

and

$$
\begin{aligned}
\int_{c}^{b} h_{2}^{2}(b-s) & v(s) P(s) d s \\
= & \int_{c}^{b}\left(\frac{h(b-s)}{\sqrt{H(b-s)}}+\sqrt{H(b-s)}\left(r(s)-\frac{v^{\prime}(s)}{v(s)}\right)\right)^{2} v(s) P(s) d s \\
= & \int_{a}^{c}\left(\frac{h(s-a)}{\sqrt{H(s-a)}}+\sqrt{H(s-a)}\left(r(2 c-s)-\frac{v^{\prime}(2 c-s)}{v(2 c-s)}\right)\right)^{2} \\
& \times v(2 c-s) P(2 c-s) d s .
\end{aligned}
$$

Thus (2.16) implies (2.14) and therefore the system (1.1) is oscillatory by Theorem 2.3. The proof of Theorem 2.5 is complete. 
Similarly, from Corollary 2.3 we obtain

TheOREM 2.6. Under the conditions of Theorem 2.5 with (2.16) replaced by

$$
\begin{aligned}
\int_{a}^{c} H(s, a) v(s)[ & \operatorname{tr} Q(s)+\operatorname{tr} Q(2 c-s)] d s \\
> & \frac{1}{4} \int_{a}^{c}[v(s) \operatorname{tr} P(s)+v(2 c-s) \operatorname{tr} P(2 c-s)] \frac{h^{2}(s-a)}{H(s-a)} d s \\
& +\frac{1}{2} \int_{a}^{c}\left[\left(r(2 c-s)-\frac{v^{\prime}(2 c-s)}{v(2 c-s)}\right) v(2 c-s) \operatorname{tr} P(2 c-s)\right. \\
& \left.-\left(r(s)-\frac{v^{\prime}(s)}{v(s)}\right) v(s) \operatorname{tr} P(s)\right] h(s-a) d s \\
& +\frac{1}{4} \int_{a}^{c}\left[\left(r(2 c-s)-\frac{v^{\prime}(2 c-s)}{v(2 c-s)}\right)^{2} v(2 c-s) \operatorname{tr} P(2 c-s)\right. \\
& \left.-\left(r(s)-\frac{v^{\prime}(s)}{v(s)}\right)^{2} v(s) \operatorname{tr} P(s)\right] H(s-a) d s
\end{aligned}
$$

the system (1.1) is oscillatory.

Now we choose

$$
k(t)=1 \quad \text { and } \quad H(t-s)=(t-s)^{\alpha} \quad \text { for } \alpha>1 .
$$

Then $H^{\prime}(t-s)=(\alpha / 2)(t-s)^{\alpha / 2-1}$. Based on the above oscillation criteria we obtain the following oscillation criteria of Kamenev's type.

Corollary 2.5. Suppose that $v \in C^{1}\left(\left[t_{0}, \infty\right),(0, \infty)\right)$ satisfies

$$
\begin{aligned}
\limsup _{t \rightarrow \infty} \lambda_{n}\left[\frac{1}{t^{\alpha-1}} \int_{l}^{t}(s\right. & -l)^{\alpha} v(s) \\
& \left.\times\left\{Q(s)-\frac{1}{4}\left(\frac{\alpha}{s-l}-\left(r(s)-\frac{v^{\prime}(s)}{v(s)}\right)\right)^{2} P(s)\right\} d s\right]>0,
\end{aligned}
$$

$\limsup _{t \rightarrow \infty} \lambda_{1}\left[\frac{1}{t^{\alpha-1}} \int_{l}^{t}(t-s)^{\alpha} v(s)\right.$

$$
\left.\times\left\{Q(s)-\frac{1}{4}\left(\frac{\alpha}{t-s}+\left(r(s)-\frac{v^{\prime}(s)}{v(s)}\right)\right)^{2} P(s)\right\} d s\right]>0,
$$

for each $l \geq t_{0}$. Then the system (1.1) is oscillatory. 
Corollary 2.6. Suppose that $v \in C^{1}\left(\left[t_{0}, \infty\right),(0, \infty)\right)$ satisfies

$$
\begin{aligned}
\limsup _{t \rightarrow \infty} \frac{1}{t^{\alpha-1}} \int_{l}^{t}(s & -l)^{\alpha} v(s) \\
\times & {\left[\operatorname{tr} Q(s)-\frac{1}{4}\left(\frac{\alpha}{s-l}-\left(r(s)-\frac{v^{\prime}(s)}{v(s)}\right)\right)^{2} \operatorname{tr} P(s)\right] d s>0, }
\end{aligned}
$$

$\limsup _{t \rightarrow \infty} \frac{1}{t^{\alpha-1}} \int_{l}^{t}(t-s)^{\alpha} v(s)$

$$
\times\left[\operatorname{tr} Q(s)-\frac{1}{4}\left(\frac{\alpha}{t-s}+\left(r(s)-\frac{v^{\prime}(s)}{v(s)}\right)\right)^{2} \operatorname{tr} P(s)\right] d s>0
$$

for each $l \geq t_{0}$. Then the system (1.1) is oscillatory.

REMARK 2.1. This paper extends to matrix systems an idea of Kong [8], who presented interval criteria for oscillation of second order ODE's. Theorems 2.1-2.6 and Corollaries 2.1-2.6 complement and improve Theorems 1-3 of [4], Theorems 1-3 of [14] and the relevant results of [11] for the matrix differential system (1.3). Further, our results complement and generalize the oscillation criterion of Kong [8] for the scalar equation (1.5), Huang [6], Philos [13] and Kamenev [7] for (1.6).

From Theorems 2.1-2.6 and Corollaries 2.1-2.6, we can deduce various explicit sufficient conditions for the oscillation of (1.1), by appropriate choice of the functions $H(t, s), k(s)$ and $v(s)$. For instance, we can choose $H(t, s)=$ $(t-s)^{\alpha}, H(t, s)=[R(t)-R(s)]^{\alpha}$, or $H(t, s)=[\log U(t) / U(s)]^{\alpha}$, or $H(t, s)=$ $\left[\int_{s}^{t}(1 / w(z)) d z\right]^{\alpha}$, etc., for $t \geq s \geq t_{0} ; k(s), v(s)$ may be chosen to be $1, s$, etc., and $\alpha>1$ is a constant, $R(t)=\int_{t_{0}}^{t} d s / u(s), U(t)=\int_{t}^{\infty} d s / u(s)<\infty$ for $t \geq t_{0}, w \in C\left(\left[t_{0}, \infty\right),(0, \infty)\right)$ with $\int_{t_{0}}^{\infty}(1 / w(z)) d z=\infty$.

EXAmple 2.1. Consider the 2-dimensional system (1.1) where

$$
P(t)=\operatorname{diag}\left(\frac{1}{t}+\frac{\cos ^{2} t}{t}, \frac{1}{t}\right), \quad r(t)=\frac{1}{t}, \quad Q(t)=\operatorname{diag}\left(q_{1}(t), q_{2}(t)\right),
$$

where

$$
\begin{aligned}
& q_{1}(t)= \begin{cases}\frac{7}{t}(t-3 n), & 3 n \leq t \leq 3 n+1, \\
\frac{7}{t}(-t+3 n+2), & 3 n+1<t \leq 3 n+2, \\
-n|\sin \pi t|, & 3 n+2<t \leq 3 n+3,\end{cases} \\
& q_{2}(t)= \begin{cases}\frac{7}{t}(t-3 n), & 3 n \leq t \leq 3 n+1, \\
\frac{7}{t}(-t+3 n+2), & 3 n+1<t \leq 3 n+2, \\
-n \pi|\sin 2 \pi t|, & 3 n+2<t<3 n+3,\end{cases}
\end{aligned}
$$

$n \in \mathbb{N}_{0}=\{0,1,2,3, \ldots\}$. For any $T>0$ there exists $n \in \mathbb{N}_{0}$ such that 
$3 n>T$. Let $a=3 n, c=3 n+1$ and $H(t-s)=(t-s)^{2}$. Choose $k(t)=1$ and $v(t)=t$; then $h(t-s)=2(t-s)$ and $r(t)=v^{\prime}(t) / v(t)$. It is easy to see that (2.16) becomes

$$
\begin{aligned}
\lambda_{2}\left[\int_{a}^{c}\left\{H(s-a) v(s) Q(s)-\frac{1}{4} \frac{h^{2}(s-a)}{H(s-a)} v(s) P(s)\right\} d s\right] \\
\quad+\lambda_{1}\left[\int_{a}^{c}\{H(s-a) v(2 c-s) Q(2 c-s)\right. \\
\left.\left.\quad-\frac{1}{4} \frac{h^{2}(s-a)}{H(s-a)} v(2 c-s) P(2 c-s)\right\} d s\right] \\
=\lambda_{2}\left[\int_{3 n}^{3 n+1}\left\{(s-3 n)^{2} \operatorname{diag}(7(s-3 n), 7(s-3 n))-\operatorname{diag}\left(1+\cos ^{2} s, 1\right)\right\} d s\right] \\
\quad+\lambda_{1}\left[\int _ { 3 n } ^ { 3 n + 1 } \left\{(s-3 n)^{2} \operatorname{diag}(7(3 n+2-s), 7(3 n+2-s))\right.\right. \\
\left.\left.\quad-\operatorname{diag}^{3 n}\left(1+\cos ^{2}(6 n+2-s), 1\right)\right\} d s\right] \\
=\int_{3 n}^{3 n+1}\left\{(s-3 n)^{2} 7(s-3 n)-\left(1+\cos ^{2} s\right)\right\} d s \\
\quad+\int_{3 n}^{3 n+1}\left\{(s-3 n)^{2} 7(3 n+2-s)-1\right\} d s \\
=\int_{3 n}^{3 n+1}(s-3 n)^{2}[7(s-3 n)+7(3 n+2-s)] d s-\int_{3 n}^{3 n+1}\left(1+\cos ^{2} s+1\right) d s \\
>14 / 3-3=5 / 3>0 .
\end{aligned}
$$

Thus (2.16) is true and therefore the system is oscillatory by Theorem 2.5. However, oscillation cannot be demonstrated by the criteria in [1-16] and other papers; moreover, in the system we have $\int_{0}^{\infty} q_{i}(t) d t=-\infty(i=1,2)$.

Acknowledgments. The author thanks Prof. James S. W. Wong and Prof. Siming Zhu for their helpful comments.

\section{References}

[1] G. J. Butler, L. H. Erbe and A. B. Mingarelli, Riccati techniques and variational principles in oscillation theory for linear systems, Trans. Amer. Math. Soc. 303 (1987), 263-282. 
[2] R. Byers, B. J. Harris and M. K. Kwong, Weighted means and oscillation conditions for second order matrix differential systems, J. Differential Equations 61 (1986), 164-177.

[3] W. J. Coles and M. K. Kinyon, Summabity methods for oscillation of linear secondorder matrix differential equations, Rocky Mountain J. Math. 24 (1994), 19-36.

[4] L. H. Erbe, Q. Kong and S. Ruan, Kamenev type theorems for second order matrix differential systems, Proc. Amer. Math. Soc. 117 (1993), 957-962.

[5] P. Hartman, On nonoscillatory linear differential equations of second order, Proc. Amer. Math. Soc. 64 (1977), 251-259.

[6] C. C. Huang, Oscillation and nonoscillation for linear second order differential equations, J. Math. Anal. Appl. 210 (1997), 712-723.

[7] I. V. Kamenev, An integral test for conjugacy for second order linear differential equations, Mat. Zametki 23 (1978), 249-251 (in Russian).

[8] Q. Kong, Interval criteria for oscillation of second-order linear ordinary differential equations, J. Math. Anal. Appl. 229 (1999), 258-270.

[9] I. S. Kumari and S. Umamaheswaram, Oscillation criteria for linear matrix Hamiltonian systems, J. Differential Equations 165 (2000), 174-198.

[10] W. T. Li and R. P. Agarwal, Interval oscillation criteria related to integral averaging technique for certain nonlinear differential equations, J. Math. Anal. Appl. 245 (2000), 171-188.

[11] F. W. Meng, J. Z. Wang and Z. W. Zheng, A note on Kamenev type theorems for second order matrix differential systems, Proc. Amer. Math. Soc. 126 (1998), 391-395.

[12] N. Parhi and P. Praharaj, Oscillation criteria for second order self-adjoint matrix differential equations, Ann. Polon. Math. 72 (1999), 1-14.

[13] C. G. Philos, Oscillation theorems for linear differential equations of second order, Arch. Math. (Basel) 53 (1989), 482-492.

[14] Q. Wang, Oscillation criteria for second order matrix differential systems, ibid. 76 (2001), 385-390.

[15] A. Wintner, A criterion of oscillatory stability, Quart. Appl. Math. 7 (1949), 115117.

[16] J. R. Yan, Oscillation theorems for second order linear differential equation with damping, Proc. Amer. Math. Soc. 98 (1986), 276-282.

Department of Mathematics

Department of Mathematics

Guangxi Normal University

Guilin, 541004, P.R. China

Zhongshan University

E-mail: yangqigui@263.net

Reçu par la Rédaction le 8.11.2001

Révisé le 26.3.2002 